\title{
Laparoscopic surgery of esophageal hiatus hernia - single center experience
}

Jacek Piątkowski, Marek Jackowski, Jacek Szeliga

Clinic of General, Gastroenterological and Oncological Surgery, Collegium Medicum, Bydgoszcz, Nicolaus Copernicus University, Torun, Poland

Videosurgery Miniinv 2014; 9 (1): 13-17 DOI: 10.5114/wiitm.2014.40174

\begin{abstract}
Introduction: Esophageal hiatal hernias are the most frequent types of internal hernias. This condition involves disturbance of normal functioning of the stomach cardiac mechanism and reflux of the gastric contents to the esophagus. Aim: To evaluate postoperative results in our Clinic and the comparison of these results to data from the literature. Material and methods: One hundred and seventy-eight patients underwent surgery due to esophageal hiatal hernia at the Clinic of General, Gastroenterological and Oncological Surgery, Collegium Medicum, Bydgoszcz, Nicolaus Copernicus University, Torun, Poland, from 2006 to 2011. All operations were performed using laparoscopy. Fundoplication by means of the Nissen-Rossetti method was carried out in 172 patients while Toupet's and Dor's methods were applied in 4 and 2 patients, respectively.

Results: Average time of the surgery was $82 \mathrm{~min}$ (55-140 min). Conversion was performed in 4 cases. No serious intraoperative complications were noted. In the postoperative period, dysphagia was reported in 20 patients (11.2\%). Postoperative wound infection was observed in 1 patient (0.56\%). Hernias in the trocar insertion area were reported in 3 patients (1.68\%). Ailments recurred in 6 patients. The recurrence of esophageal hiatal hernia was confirmed in 2 patients. Patients with recurrent hernia were re-operated using a laparoscopic approach.

Conclusions: Laparoscopic surgery is a simple and effective approach for patients with gastroesophageal reflux symptoms due to diaphragmatic esophageal hiatus hernia. The number of complications is lower after laparoscopic procedures than after "open" operations.
\end{abstract}

Key words: laparoscopy, hiatal hernia, fundoplication.

\section{Introduction}

Esophageal hiatal hernias are the most frequent types of internal hernias. This is a condition involving protrusion of the stomach into the chest. Over $90 \%$ of esophageal hernias are sliding hernias while about $10 \%$ are periesophageal and mixed hernias. Therefore, esophageal hiatal hernia is identified as a sliding hernia.

This condition involves disturbance of normal functioning of the stomach cardiac mechanism and reflux of the gastric contents to the esophagus [1-4].
During recent years, this disease unit has attracted more attention and numerous papers have been published on gastroesophageal reflux and the changes defined as Barrett's esophagus. There is much interest in gastroesophageal reflux disease (GERD) due to frequent occurrence of this condition, affecting almost $40 \%$ of the population of highly industrialized countries $[5,6]$.

Gastroesophageal reflux refers to all symptoms and/or morphological changes within the esophagus due to the reflux of stomach acid. Numerous papers

\section{Address for correspondence}

Jacek Szeliga MD, PhD, Clinic of General, Gastroenterological and Oncological Surgery, Collegium Medicum, Nicolaus Copernicus University, 53/59 Św. Józefa St, 87-100 Torun, Poland, phone: +48 509207 072, e-mail: jacky2@wp.pl 
also indicate the risk of such changes due to duodenal-gastro-esophageal reflux and the reflux of the duodenal contents to the esophagus $[1,4]$.

Symptoms characteristic for this disease unit have been known for many years, although its pathogenesis has not been fully explained. Esophageal hiatal hernia is known as one of the causes of such symptoms, responsible for the flow of stomach contents to the esophagus. Obviously, these ailments are not presented in all patients; however, the comparison of morphological changes in the esophageal mucosa with subjective complaints and diaphragmatic esophageal hiatal hernia definitely indicates that hernia is the main problem in this condition $[2,7,8]$.

Another issue concerns changes in the esophageal mucosa, referred to as Barrett's syndrome. In this condition, the normal squamous epithelium lining of the esophagus is replaced by columnar epithelium. In most cases, this is due to chronic reflux of stomach contents to the esophagus and the related chronic inflammatory changes. Columnar epithelium is typically a mixture of different cells and glandular structures, resembling the elements of normal gastric or intestinal mucosa. The next stage involves the development of morphological dysplastic changes within this epithelium. Dysplasia in Barrett's epithelium ranges from minor to carcinoma "in situ". It is generally believed that Barrett's esophagus is a precancerous condition. Since the 1970s, a constant increase in the number of patients with Barrett's esophagus has been reported. This can be probably attributed to long-term use of medications, resulting in a decrease in gastric juice acidity, but also with more frequent access to endoscopy and familiarity with changes related to Barrett's esophagus among physicians.

Once Barrett's esophagus is diagnosed, prompt antireflux surgery is recommended to stop the inflammatory process in the esophagus caused by reflux and to stop progression to dysplasia.

Surgical treatments of esophageal hiatus hernias have been performed for many years; however, the introduction of laparoscopic techniques to surgery decreased GERD-related ailments, shortened the period of hospitalization and convalescence, and increased the number of patients suffering from GERD-related conditions who decide to have surgical treatment.

Unfortunately, postoperative treatment involves the risk of complications. These can be classified into intraoperative and postoperative complications.
The former include complications related to Veress needle insertion, development of peritoneal edema, bleeding due to vascular damage, damage to other organs and the development of pleural or mediastinal edema. The latter include dysphagia, infection of postoperative wounds, hernias at the sites of trocar catheter insertion, recurrent hernia, ischemia or migration of gastric cuff, narrowing of the cardia and inability to burp and/or vomit.

\section{Aim}

The aim of this study was to evaluate postoperative results in our Clinic and the comparison of these results to data from the literature.

\section{Material and methods}

One hundred and seventy-eight patients underwent surgery due to esophageal hiatal hernia at the Clinic of General, Gastroenterological and Oncological Surgery, Collegium Medicum, Bydgoszcz, Nicolaus Copernicus University, Torun, Poland, from 2006 to 2011. The sample included 103 males and $75 \mathrm{fe}$ males. The mean age of the patients was 47.6 years (19-76 years).

The clinic has no equipment for 24-hour $\mathrm{pH}$-metry or esophageal manometry, and patients with hiatus hernia of the esophagus experiencing gastroesophageal reflux symptoms were qualified for surgical treatment.

The patients qualified for surgery had symptoms of gastroesophageal reflux which persisted after conservative treatment or quickly recurred after withdrawal of medications, or were diagnosed with progression of inflammatory changes revealed during gastroscopic tests despite no subjective complaints.

Gastrofiberoscopy and X-ray of the upper segment of the alimentary tract with contrast medium were performed in all patients prior to the surgery. The endoscopy assessed the size of hiatus hernia of the esophagus and inflammatory changes of the esophageal mucosa. Type I hiatus hernias were the most common type (170/178). The inflammatory changes were assessed according to the four-grade scale of reflux esophagitis, established in Los Angeles. In 46 cases, endoscopy revealed grade $A$, in 62 grade $B$, while grades $C$ and $D$ were found in 47 and 23 patients, respectively. X-ray of the upper alimentary tract revealed diaphragmatic esophageal hiatal hernia in all patients. 


\section{Results}

All operations were performed using laparoscopy. All patients had their hernial sac removed from the mediastinum, all diaphragmatic branches were sewn up and fundoplication was carried out. Esophageal hiatal hernia was narrowed using three sutures, by joining diaphragmatic branches above the raised esophagus. No meshes were used for hernioplasty.

Fundoplication by means of the Nissen-Rossetti method was carried out in 172 patients while Toupet's and Dor's methods were applied in 4 and 2 patients, respectively. Drains were routinely left in the fundoplication area; the drains were usually removed on the second day postoperatively. During hernia preparation surgery, there were problems reported with the preparation of hernial sac and stomach removal from the chest, although the fundus and part of the body of the stomach were shifted to the chest in several cases.

Average time of the surgery was $82 \mathrm{~min}$ (55$140 \mathrm{~min})$. In each case, drains were left in the fundoplication area and removed on the second day postoperatively. No serious intraoperative or postoperative complications were noted.

Conversion was performed in 4 cases - in 3 cases due to problems with the identification of anatomical structures (because of substantial obesity and the amount of adipose tissue in 1 case, while in the two remaining cases, it was performed due to adhesions after the procedures within the epigastrium). In the $4^{\text {th }}$ case during the preparation of the esophagus and diaphragmatic branches, subdermal emphysema and mediastinal edema accompanied by a decrease in arterial blood pressure were observed in the fourth patient. Conversion to the open method was the approach of choice after desufflation and laparotomy, including Nissen fundoplication, and diaphragmatic branches were sewn up. Blood pressure returned to normal and substantial absorption of the subdermal emphysema on the neck was noted. Within several hours postoperatively, total absorption of the air from the subdermal tissue of the neck was observed.

The average time of hospitalization at the clinic was 3 days (2-5 days). Regression of symptoms was observed in all patients after the surgery. No serious intraoperative complications were noted.

In 4 cases, pleural edema developed due to pleural rupture during removal of the hernial sac from the mediastinum. The pleura was sewn up in all patients. After the procedure, chest X-ray was performed. No patient required pleural drainage. Subdermal emphysema and mediastinal edema developed in one patient, which spontaneously abated after desufflation.

In 1 case, the frontal esophagus wall was damaged during hernia preparation. The damage was sewn up using two layers of sutures and covered with fundoplication. No postoperative complications were noted in this patient.

The perioperational mortality rate was $0 \%$ in the studied sample.

In the postoperative period, dysphagia was reported in 2 patients (11.2\%), but it subsided spontaneously in 16 patients within 2 months postoperatively. Dysphagia persisted up to 6 months in 4 patients. These patients underwent gastroscopic examination and endoscopic dilation of the gastric cardia with a pneumatic balloon. Postoperative wound infection was observed in one patient (0.56\%). Hernias in the trocar insertion area were reported in 3 patients (1.68\%). Ailments recurred in 6 patients. The recurrence of esophageal hiatal hernia was confirmed (computed tomography scan revealed shift of the gastric cuff to the chest in 2 patients). Nine patients (5.05\%) required secondary surgical intervention. Three patients were reoperated due to hernias. Four patients required surgical treatment due to recurrent esophageal hernias in the trocar insertion area, and 2 patients were re-operated on because of gastric cardia stenosis since the diaphragmatic branches were too tightly sewn up (no improvement after endoscopic dilation of the gastric cardia).

Patients with recurrent hernia were re-operated on using a laparoscopic approach to sew up the diaphragmatic branches. Patients with gastric cardia stenosis had their esophageal hiatus widened. In 1 of the female patients, the hernia recurred after 6 months; the patient underwent re-operation - hernia plasty was performed, as well as anterior fundoplication using Dor's approach.

\section{Discussion}

According to various data, patients having gastroesophageal reflux symptoms with esophageal hiatus hernia being the reason of complaints constitute $60-80 \%$ of all patients with $\operatorname{GERD}[1,4,6,9]$. Reflux may also be due to other reasons; however, 
because of no appropriate equipment at the clinic, patients with coexisting symptoms of reflux and diaphragmatic esophageal hernia were qualified for surgery.

Patients with reflux frequently undergo long-term conservative treatment, sometimes without any significant improvement. Stomach content reflux to the esophagus results in changes within the esophageal mucosa; the untreated condition may lead to changes in the esophageal mucosa and the development of Barrett's esophagus or, in consequence, to esophageal cancer. There are also reports saying that longterm use of medications reducing gastric secretion in patients with reflux, particularly proton pump blockers, may favor the development of esophageal cancer.

Therefore, patients with GERD requiring longterm treatment or showing no clear improvement despite treatment, especially those with concomitant esophageal hiatus hernia, should be qualified for surgical treatment.

The percentage of complications after esophageal hiatal hernia surgery performed at medical centers is very low. According to literature data, good results are obtained in 90-95\% of cases [4, 8, 10-12]. Postoperative complications are rare and appropriate training in using laparoscopic techniques allows them to be avoided.

Dysphagia is the most frequent postoperative complication [13]. According to literature data reviewed, its incidence ranges from $5 \%$ to $30 \%$. Adequate mobilization of the gastric fundus allows for fundoplication without tension [7, 11, 14]. This prevents dysphagia caused by cuff compression of esophageal torsion. Sewing up the branches also significantly contributes to the reduction of the risk of dysphagia. If dysphagia persists longer than one month, endoscopic examination is recommended to exclude stenosis of the gastric cardia.

Hernias rarely develop at trocar insertion sites (1-3\%) [11]. Adequate sewing of the wounds after trocar insertion prevents this complication. Obesity is a frequent risk factor for development of this complication. Surgical treatment is usually required in such cases. Hernia is reported to recur in $7-20 \%$ of patients. Total excision of the hernial sac reduces risk of recurrence. According to the analyzed data, synthetic meshes do not reduce the risk of hernia recurrence [15]. Hernia recurrence is sometimes accompanied by migration of the gastric cuff to the mediastinum. In the majority of patients, ailments related to gastroesophageal reflux recur. These patients are qualified for surgical treatment.

Inability to burp and/or vomit is a frequent postoperative symptom. Usually patients accept this complication and do not require further treatment.

In the studied sample, the number of complications did not differ from literature data. The number of complications is smaller than that observed after open operations $[7,11]$.

All advantages resulting from adopting the minimally invasive approaches are maintained (shorter hospitalization period, better cosmetic effects, less pain, quicker return to full fitness).

\section{Conclusions}

Laparoscopic surgery is a simple and effective approach for patients with gastroesophageal reflux symptoms due to diaphragmatic esophageal hiatus hernia. The number of complications is lower after laparoscopic procedures than after "open" operations.

\section{References}

1. Stanowski E. Gastroesophageal disease - indications and methods of surgiacl treatments [Polish]. Videochirurgia 1998; 1: 6-11.

2. Stanowski E. Laparoscopic antireflux surgery - the choice of treatment - own experience [Polish]. Pol Przegląd Chir 2001; 73: 919-23.

3. Perdikis G, Hindre RA, Lund RJ. Laparoscopic Nissen fundoplication. Where do we stand? Surg Laparosc Endoscopy 1997; 7; 17-21.

4. Tarnowski W, et al. Laparoscopic surgery in the treatment of gastroesophageal reflux [Polish]. Pol Przegląd Chir 2001; 73: 924-31.

5. Peters JH, De Meester TR. Indications, principles of procedure selection and technique of laparoscopic Nissen fundoplication. Seminars Laparosc Surg 1995; 2: 27-44.

6. Peters JH, De Meester TR. Indications, benefits and outcome of laparoscopic Nissen fundoplication. Dig Dis 1996; 14: 169-79.

7. Watson DI, de Beaux AC. Complications of laparoscopic antireflux surgery. Surg Endosc 2001; 15; 344-52.

8. Dallemagne B, Weerts J, Markiewicz S, Dewandre JM. Clinical results of laparosocpic fundoplication at ten years after surgery. Surg Endosc 2006; 20: 159-65.

9. Frantzides R, Carlson MA. Laparoscopic versus conventional fundoplication. J Laparoendosc Surg 1995; 5: 137-43.

10. Granderath FA, Kamolz T, Schweiger UM, Pasiut M. Long-term results of laparoscopic antireflux surgery. Surg Endosc 2002; 16: 753-57.

11. Rathore MA, Andrabi SI, Bhatti MI, et al. Metaanalysis of recurrence after laparoscopic repair of paraoesophageal hernia. JSLS 2007; 11: 456-60. 
12. Nguyen NT, Christie C, Masoomi H, et al. Utilization and outcomes of laparoscopic versus open paraoesophageal hernia repair. Am Surg 2011; 77: 1353-7.

13. Hunter JG, Swanstrom L, Waring JP. Dysphagia after laparoscopic antireflux surgery. Ann Surg 1996; 224: 51-7.

14. Granderath FA, Schweiger UM, Kamolz T, Pointner R. Dysphagia after laparoscopic antireflux surgery: a problem of hiatal closure more than a problem of the wrap. Surg Endosc 2005; 19 : 1439-46.

15. Pallabazer G, Santi S, Parise P, Solito B. Giant hiatal hernias: direct hiatus closure has an acceptable recurrence rate. Updates Surg 2011; 63: 75-81.

Received: 5.02.2013, accepted: 16.05.2013. 